\title{
Elaboración de un Programa Computacional Especializado para Hidráulica de Canales hidrov 1.0
}

RESPONSABLE: Ing. Msc. Edwin Martin Pino Vargas

\begin{abstract}
RESUMEN. La estructura del programa estará basado en el desarrollo de las aplicaciones de la hidráulica de canales; en tal sentido, establecemos el uso de MATLAB, que es la abreviatura de MATrix LABoratory (laboratorio de matrices). Se trata de un software matemático muy versátil que ofrece un entorno de desarrollo integrado (IDE) con un lenguaje de programación propio (lenguaje M). Está disponible para las plataformas Unix, Windows y Apple Mac OS $X$. Se efectuaron aplicaciones de la hidráulica de canales en los siguientes estados: Crítico, Uniforme, Gradualmente Variado, Rápidamente Variado $y$ Espacialmente Variado, para diferentes secciones prismáticas utilizadas en la hidráulica de canales.
\end{abstract}

\begin{abstract}
The structure of the programm is based in the develop of the applications of the hydraulic water lines, at this way we set up the use of MATLAB that is the abbreviation of MATRIX LABORATORY.Its about a mathematiacian software so versatile that offers an integrated develop (IDE). With a language of the own programming. Is avalible for the platforms Unix, Windows y Apple Mac OS X. We make applications of the hydraulic water lines in some states: Critical, standard, gradually varied, rapidly varied and spatially varied, for for different prismatic sections used in hydraulic wáter lines.
\end{abstract}

INTRODUCCIÓN. Los datos hidrológicos frecuentemente requieren ser utilizados para el diseño de estructuras hidráulicas ya sea con fines de utilizacióno de previsión ante desastres naturales. Dichos datos hidrológicos son normalmente de registros extensos y de naturaleza muy variable, por lo que antes de ser utilizados requieren pasar por un estricto control estadístico, a lo que le denominamos análisis de homogeneidad o consistencia, y luego el análisis de frecuencias hidrológicas, con lo cual quedan expeditos para ser modelados. Esta aplicación fue desarrollada por el autor en el DESARROLLO DE UN PROGRAMA COMPUTACIONAL ESPECIALIZADO PARA INGENIERÍA HIDRÁULICA E HIDROLÓGICA, presentado como resultado de la investigación en el año 2007.

En la siguiente etapa, el diseñador hidráulico requiere de un programa de cómputo para ensayar diferentes alternativas de cálculo. Dicho programa será elaborado en MATLAB, con la finalidad de contar con una herramienta ágil y versátil para el procesamiento, análisis y modelación de escenarios. La elaboración de este programa se da en el marco de las exigencias académicas y prácticas que se presentan en la Ingeniería Hidráulica e Hidrológica.

\section{Soporte Teórico}

Aspectos Generales

El programa "hidro v 1.0" ha sido concebido por el autor como una necesidad del diseñador hidráulico, en el afán de tratar de cubrir la mayor cantidad de escenarios de diseño, es decir, ensayar la mayor cantidad de alternativas para logra la mejor alternativa de diseño.

\section{Soporte Matemático}

El soporte matemático requerido o necesario para elaborar el presente programa radica en la aplicación de los métodos numéricos, básicamente en lo que concierne a la solución numérica de ecuaciones de la forma $\mathrm{f}(\mathrm{x})=0$, con la aplicación del método de Nuewton Raphson, diferenciación numérica, integración numérica, ecuaciones diferenciales ordinarias con el método Runge Kutta y ecuaciones diferenciales parciales con las diferencias finitas.

\section{Soporte Computacional, Uso De Matlab}

MATLAB es la abreviatura de MATrix LABoratory (laboratorio de matrices). Se trata de un software matemático muy versátil que ofrece un entorno de desarrollo integrado (IDE) con un lenguaje de programación propio (lenguaje M). Está disponible para las plataformas Unix, Windows y Apple Mac OS X.

Entre sus prestaciones básicas se hallan: la manipulación de matrices, la representación de datos y funciones, la implementación de algoritmos, la creación de interfaces de usuario (GUI) y la comunicación con programas en otros lenguajes y con otros dispositivos hardware. El paquete MATLAB dispone de dos herramientas adicionales que expanden sus prestaciones, a saber: Simulink (plataforma de simulación multidominio) y GUIDE (editor de interfaces de usuario - GUI). Además, se pueden ampliar las capacidades de MATLAB con las cajas de herramientas (toolboxes); y las de Simulink con los paquetes de bloques (blocksets). 
Es un software muy usado en universidades y centros de investigación y desarrollo. En los últimos años ha aumentado el número de prestaciones, como la de programar directamente procesadores digitales de señal o crear código VHDL.

Fue creado por The MathWorks en 1984, surgiendo la primera versión con la idea de emplear paquetes de subrutinas escritas en Fortran en los cursos de álgebra lineal y análisis numérico, sin necesidad de escribir programas en dicho lenguaje. El lenguaje de programación M fue creado en 1970 para proporcionar un sencillo acceso al software de matrices LINPACK y EISPACK sin tener que usar Fortran.

En 2004, se estimaba que MATLAB era empleado por más de un millón de personas en ámbitos académicos y empresariales. Finalmente, MATLAB es un programa de cálculo numérico orientado a matrices. Por tanto, será más eficiente si se diseñan los algoritmos en términos de matrices y vectores.

\section{Descripción y Desarrollo del Programa, Aplicaciones}

La estructura del programa estará basada en el desarrollo de las aplicaciones de la hidráulica de canales; en tal sentido, establecemos la siguiente estructura presentada por etapas.

\section{Desarrollo del Programa: Pantalla Principal}

Esta pantalla principal ha sido elaborada en el mismo editor del MATLAB, es decir, como un archivo "m", simplemente. Cabe destacar que se puede hacer una programación en el ambiente gráfico generando archivos tipo "fig".

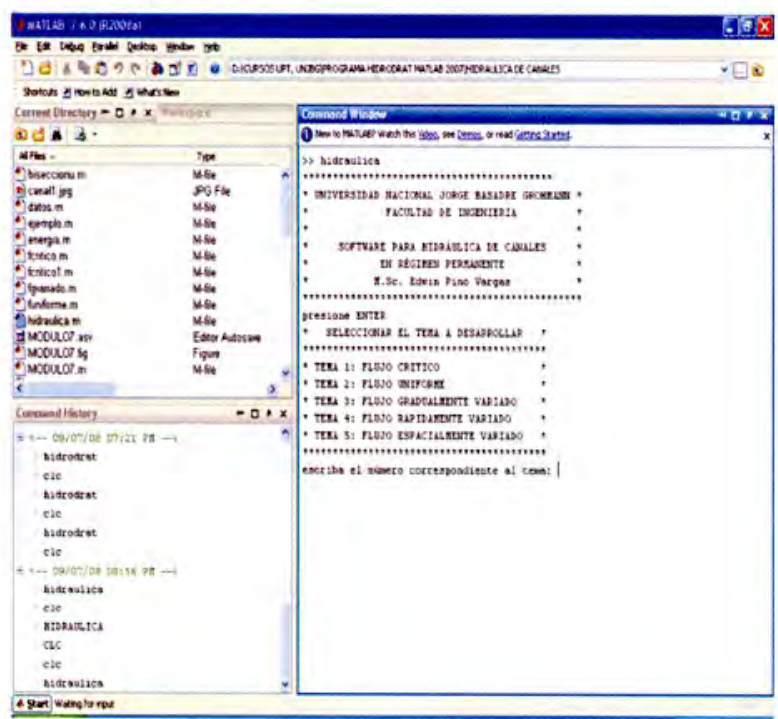

La codificación en MATLAB de esta pantalla es la siguiente, la misma que ha sido almacenada en un archivo tipo "m":

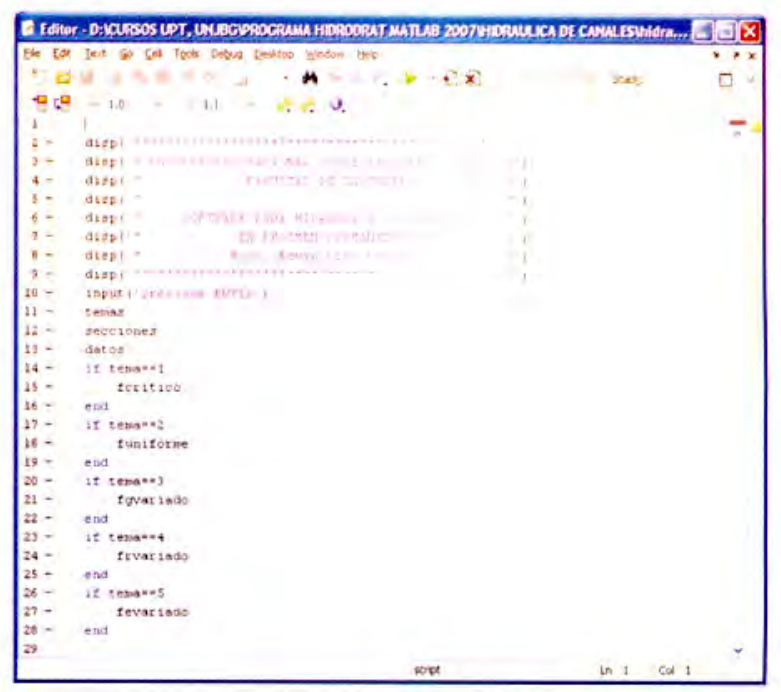

También se puede utilizar un archivo tipo "fig", generado en el módulo guide del MATLAB. En este caso podemos presentar una pantalla gráfica más amigable, y de fácil acceso al usuario.

\section{Flujo Crítico}

Se define para un flujo turbulento, completamente desarrollado e incompresible, la energía específica como la suma de los términos de carga cinética más carga potencial:

$$
E=\mathrm{y}+\begin{gathered}
<\mathrm{v}>^{2} \\
2 \times g
\end{gathered}
$$

Donde y es la profundidad del flujo, normal al lecho del canal. En términos del caudal, $(\mathrm{Q}=<\mathrm{v}>\mathrm{A})$ :

$$
E=\mathrm{y}+\underset{2 \times g \times A^{2}}{Q^{2}}
$$

Para un caudal dado, la energía específica posee un mínimo. Este punto corresponde a la profundidad crítica $\left(\mathrm{y}_{\mathrm{CR}}\right)$, procedimiento para encontrar $\mathrm{y}_{\mathrm{CR}}$ : expresar el área (A) en función de la profundidad (y). Derivar la ecuación de energía especifica respecto de y. Hacer la ecuación derivada igual a cero despejar $\mathrm{y}_{\mathrm{CR}}$.

\section{Codificación Matlab}

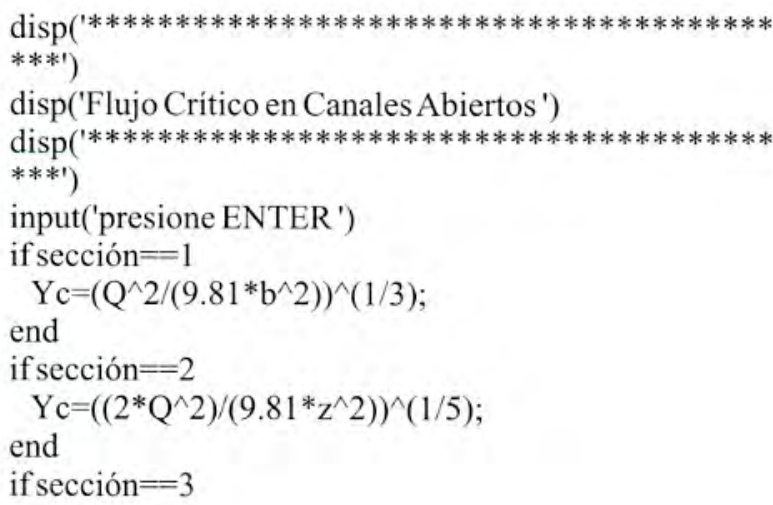




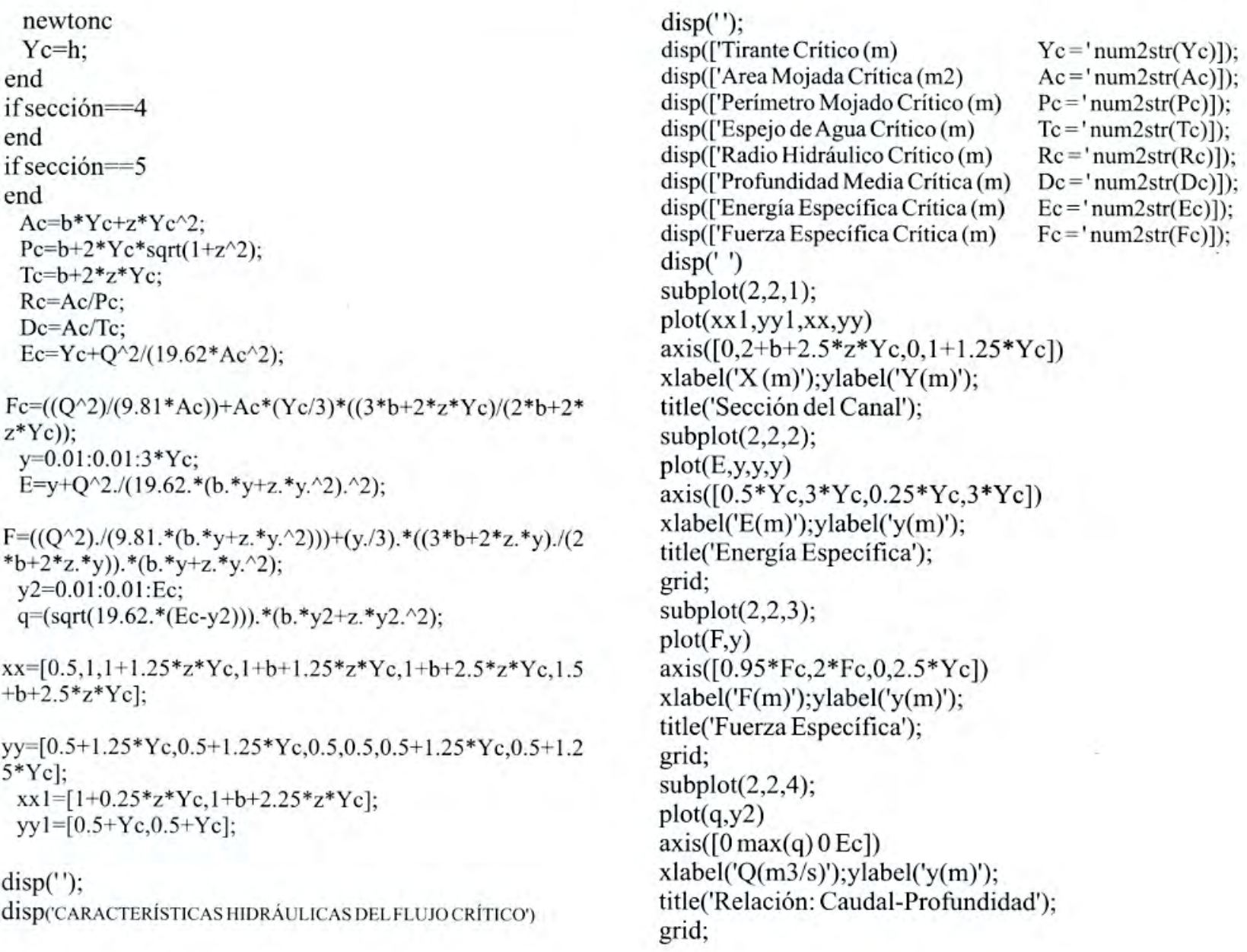

\section{Corriendo el Programa en Matlab}

Se muestran la pantalla principal, donde se selecciona la opción de canal rectangular y flujo crítico:

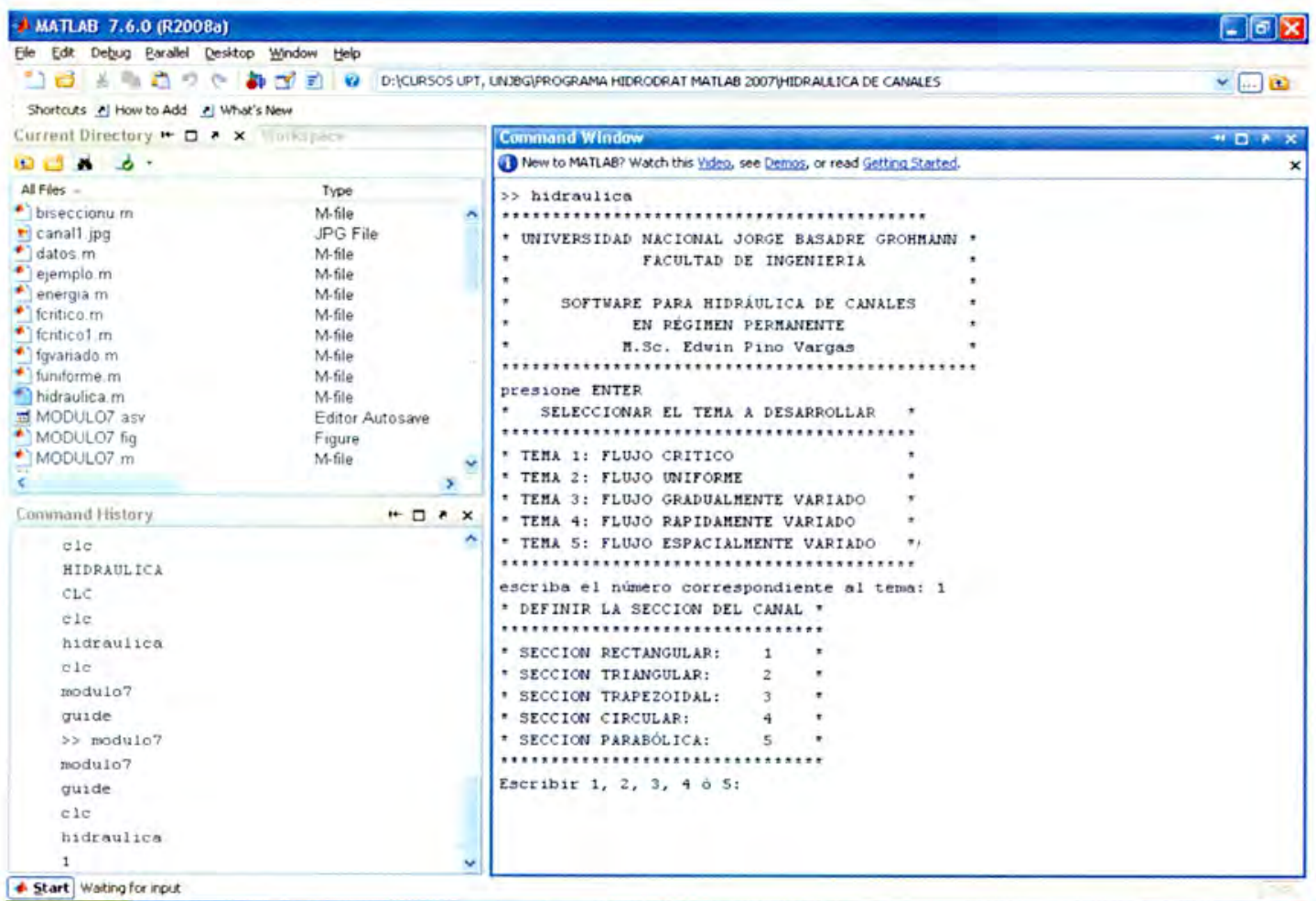


Para unos valores arbitrarios asumidos de $5 \mathrm{~m} 3 / \mathrm{s}$ de caudal y un ancho de solera de $2 \mathrm{~m}$, se tienen los siguientes resultados de salida del programa, los mismos que han sido verificados manualmente y validados.

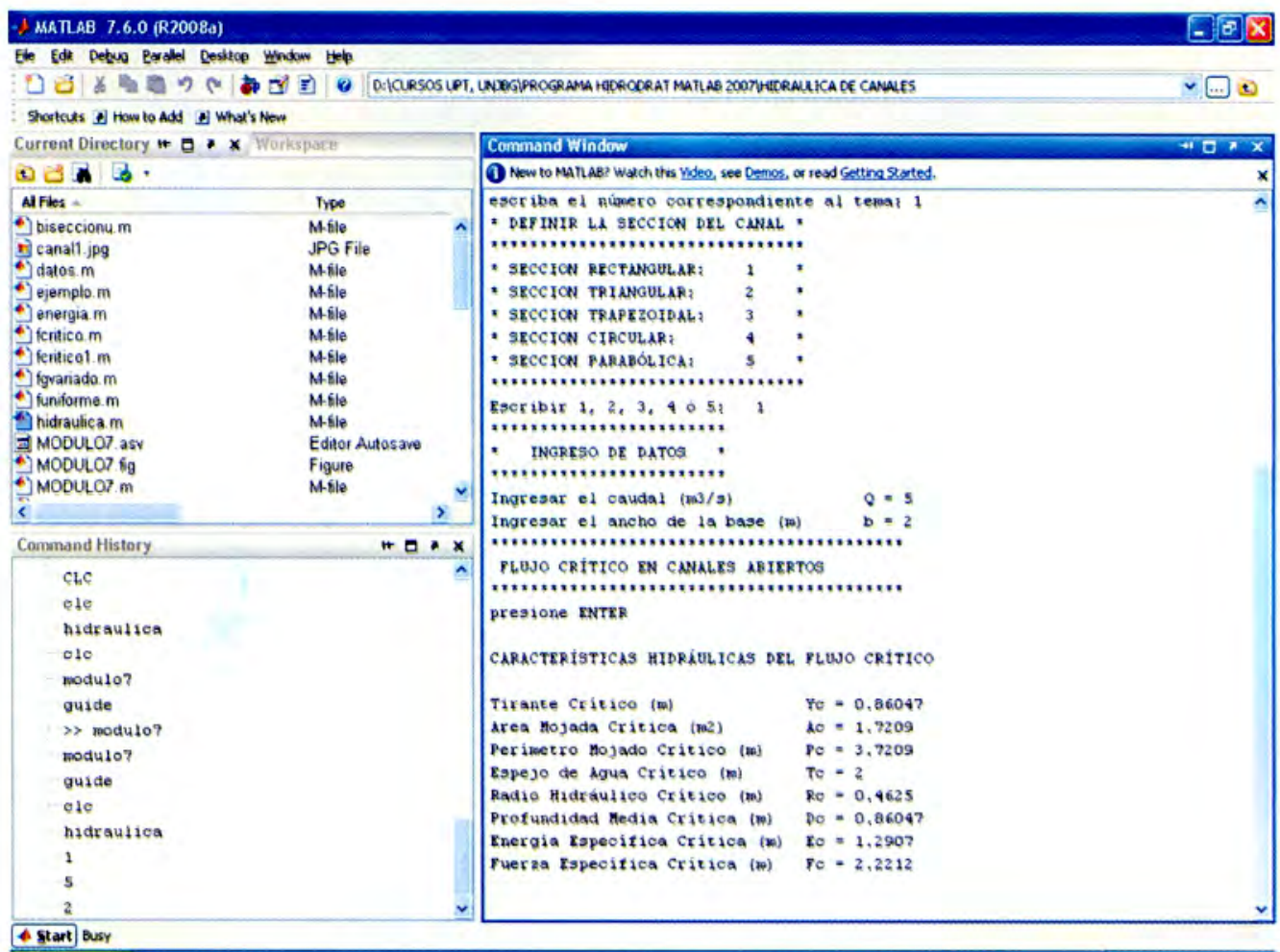

Adicionalmente, se generan curvas importantes en el flujo a superficie libre, las mismas que se muestran a continuación:

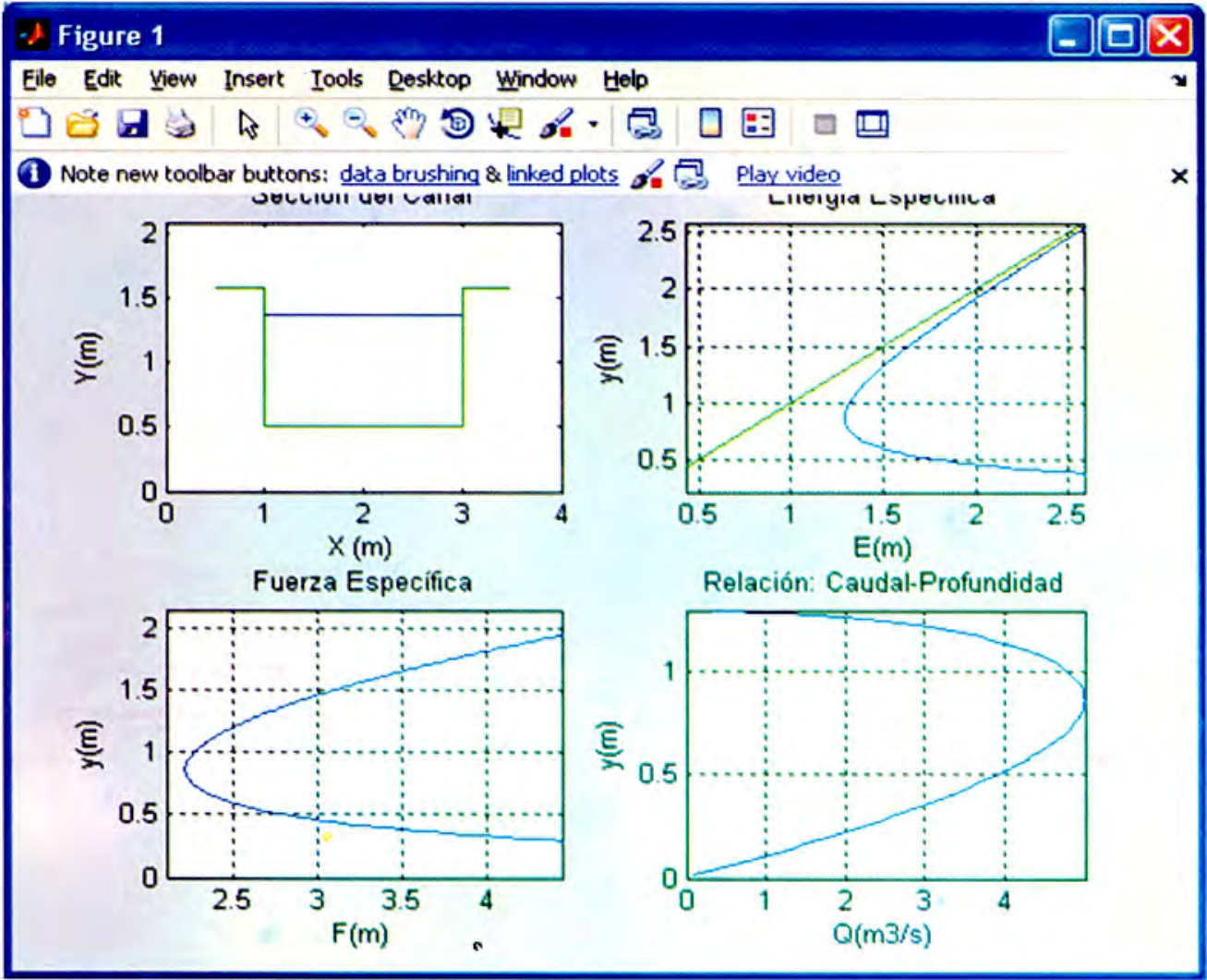


En el caso del flujo uniforme se tiene la misma concepción:

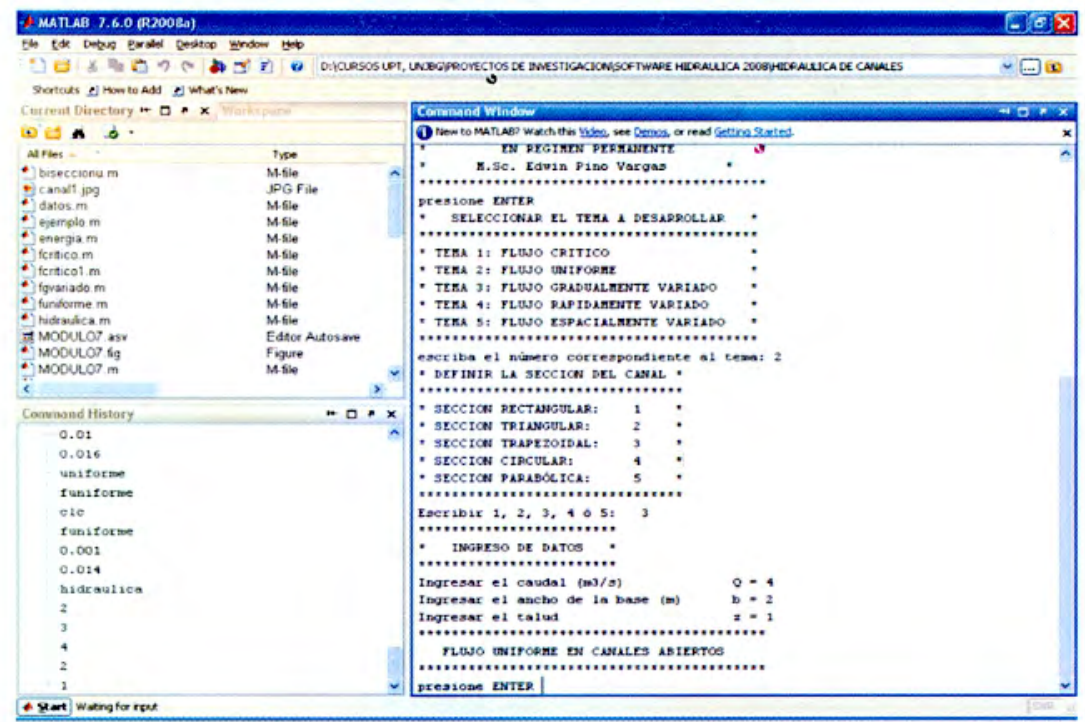

\section{Eimantas 7.6.0 (R20080)}

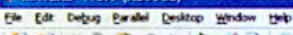

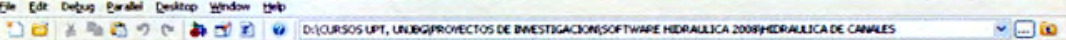

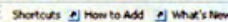

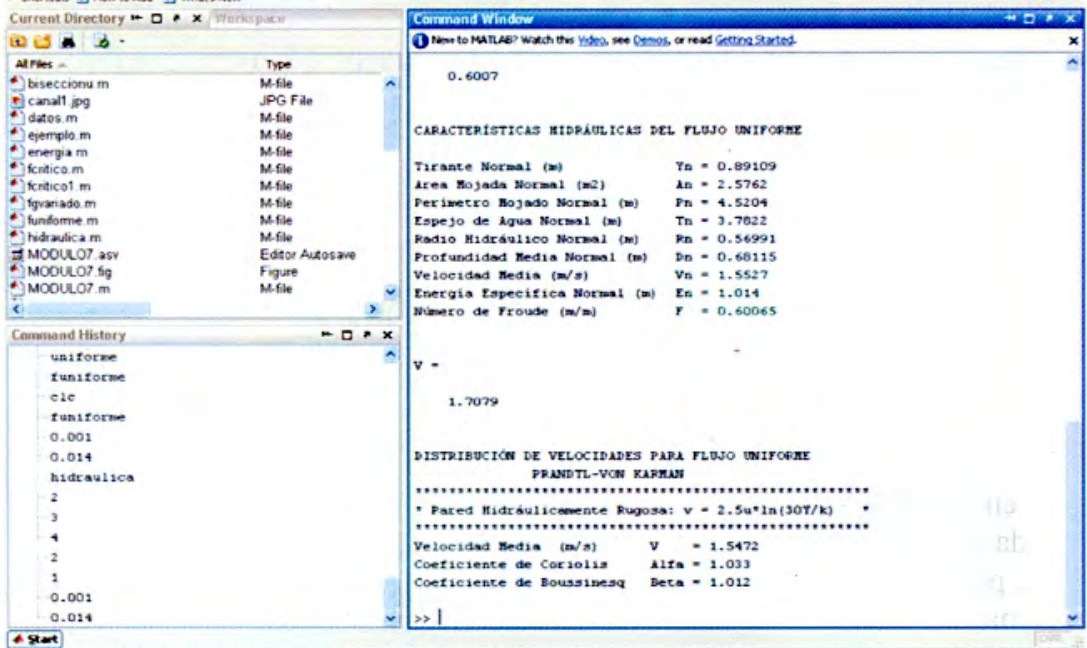
seser

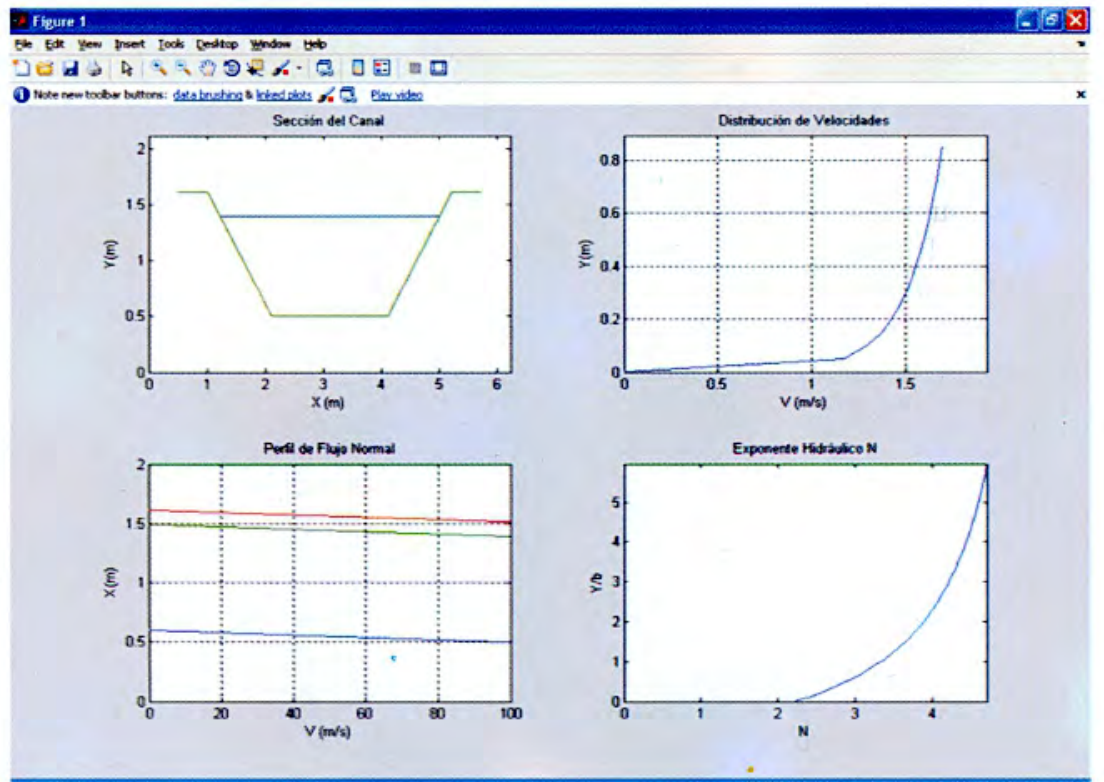




\section{VANCE DE APLICACIONES ENTORNO FIG DE MATLAB}

En este caso se ha desarrollado una pantalla gráfica, muy amigable y que cumpla el objetivo de interacción entre programa y usuario, para las diferentes aplicaciones realizadas en el presente trabajo.

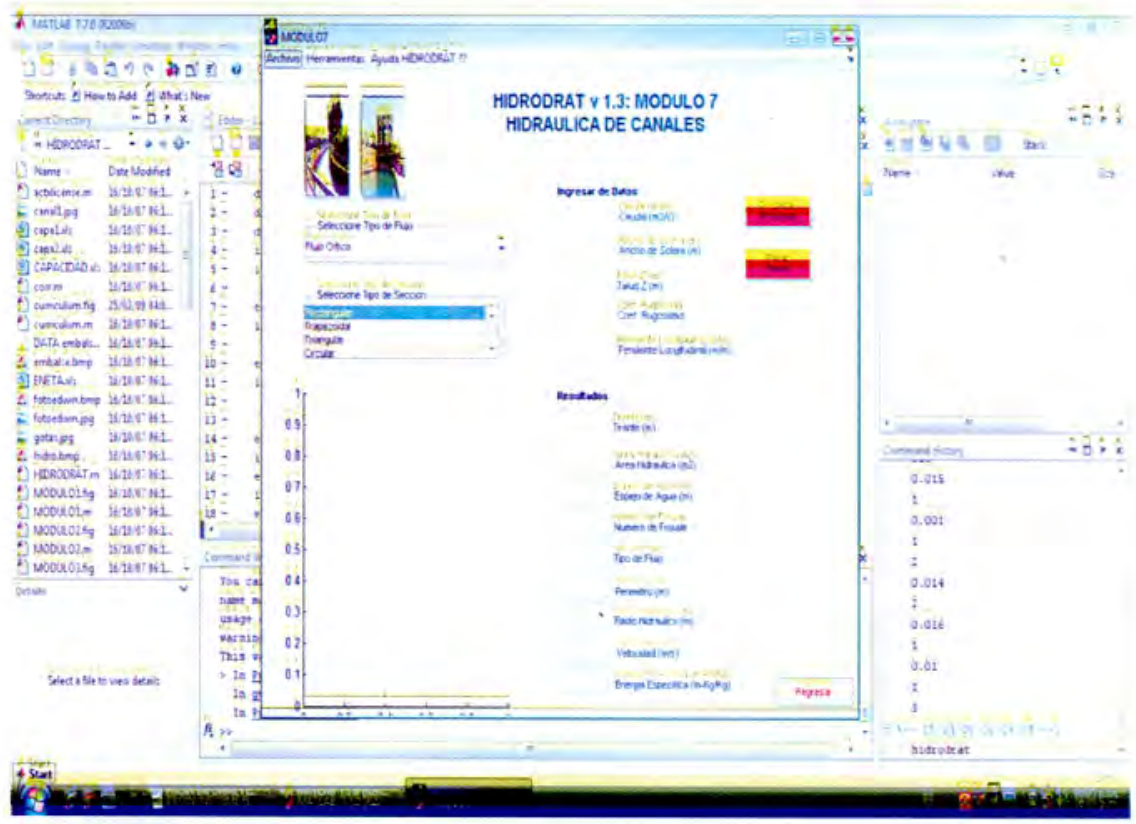

En esta pantalla se puede observar que el programa tiene una gran capacidad de interacción con el usuario, de tal forma que se puede elegir el tipo de flujo, el tipo de sección hidráulica y luego especificar los datos de entrada, para poder obtener los respectivos resultados, tabulados como cálculos y algún tipo de gráfico que sea representativo del proceso calculado.

\section{CONCLUSIONES}

Luego del desarrollo del presente trabajo de investigación, se ha podido llegar a las siguientes conclusiones:

1. La programación en MATLAB, resulta muy práctica, simplificada, y objetiva, lo cual facilita mucho el trabajo de programación y simplifica el listado de los programas.

2. Se logro desarrollar y describir los procesos físicos y empíricos de la hidráulica de canales abiertos, en forma detallada, para luego especificar el ecuacionamiento respectivo.

3. Una vez que estaba especificado el ecuacionamiento del proceso hidráulico, se procedió a la codificación en MATLAB, utilizando una serie de algoritmos numéricos como es el caso del Algoritmo de Newton Raphson, uno de los más conocidos y aplicados en esta materia.

4.Asimismo se utilizó diferenciación numérica, integración numérica, ecuaciones diferenciales ordinarias con el método Runge Kutta.0

\section{RECOMENDACIONES}

Se recomiendo lo siguiente:

1. La recomendación principal es que se dé continuidad con el trabajo desarrollado; en esta etapa se han logrado aportes muy importantes, pero es necesario continuar con dicho trabajo a fin de completar y mejorar la presentación del programa.

2. Integrar el entorno gráfico FIG de MATLAB con los programas fuentes *.m, desarrollados en el presente trabajo de investigación.

\section{REFERENCIAS BIBLIOGRÁFICAS}

Máximo VILLÓN B. 1995. Hidráulica de Canales. Ed. Tecnología de Costa Rica.

LINSLEY KOHLER. Hidrología para Ingenieros. Ed. McGraw Hill Book Co.

Ven TE CHOW Editor. Handbook of Aplied Hydrology. Ed. McGraw Hill Book.

G. MONSALVE SÁENZ. 1995. Hidrologia en la Ingeniería. Ed. Escuela Colombiana de Ingeniería.

Ven TE CHOW, David R. Maidment, Larry. Mays. 1994. Hidrologia Aplicada. Mc.Graw Hill.

MEJÍA MARCA cuzco Abel. 1994. Métodos Estadísticos en Hidrología. La Molina, Lima.

Separatas del Curso de Hidrología. Edwin PINO VARGAS 2003. 